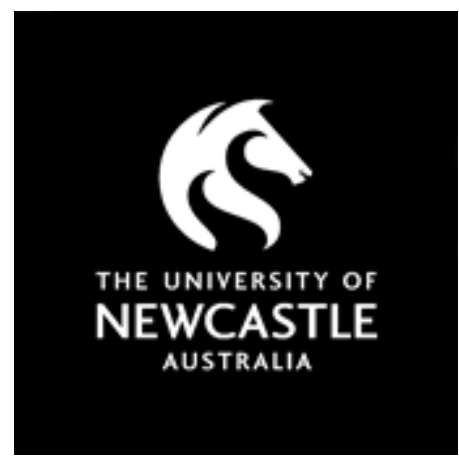

NOVA

University of Newcastle Research Online

nova.newcastle.edu.au

Skinner, Geoff; Fletcher, Richard; May, Chris. "An ICT eHealth mobile web application for young indigenous fathers - StayinOnTrack", Published in Proceedings of the IEEE International Conference on Multimedia \& Expo Workshops (ICMEW) 2017 (Hong Kong, China 10-14 July, 2017) p. 103-108. (2017)

Available from: http://dx.doi.org/10.1109/ICMEW.2017.8026252

(C) 2017 IEEE. Personal use of this material is permitted. Permission from IEEE must be obtained for all other uses, in any current or future media, including reprinting/republishing this material for advertising or promotional purposes, creating new collective works, for resale or redistribution to servers or lists, or reuse of any copyrighted component of this work in other works.

Accessed from: http://hdl.handle.net/1959.13/1352230 


\section{An ICT eHealth Mobile Web Application for Young Indigenous Fathers - StayinOnTrack}

\author{
Geoff Skinner \\ School of Electrical Engineering and Computing \\ Faculty of Engineering \\ The University of Newcastle \\ Newcastle, Australia
}

\author{
Richard Fletcher \& Chris May \\ Family Action Centre \\ Faculty of Health and Medicine \\ The University of Newcastle \\ Newcastle, Australia
}

\begin{abstract}
The targeted use of Information and Communication Technologies (ICT) can provide a very effective holistic platform for the delivery of eHealth services. Through the integration of communication information delivery mediums such as SMS IMMS coupled with the web andlor native mobile applications information technologies, eHealth services can be extended and readily adapted to mobile devices giving rise to the domain of mHealth (mobile health). This paper details an innovative new platform for providing mental health and support services to its registered users. More specifically the demographical focus is on young indigenous Australian men, providing health service and support for those that are soon to be or new fathers. The research and body of work represents an implemented ICT framework that utilizes SMS, MMS, email, and a companion mobile web application collectively entitled StayinOnTrack. The implemented system has now been in production for 18 months with an active pilot community user base. As the results have been successfully positive and encouraging, the project is undergoing nationwide expansion.
\end{abstract}

Keywords-eHealth; mHealth; Mobile Web Applications; Mobile Communications; Multimedia Applications

\section{INTRODUCTION}

mHealth has opened up a vast array of opportunities and research to deliver quality health services to mobile devices all over the world. Essentially, all that is required is a mobile device (preferably a smart phone or tablet) with some form of communication connection, whether that is $\mathrm{WiFi}$ or integrated mobile telecommunications. In Australia, mobile connections come in various forms with the current highest available bandwidth being referred to as $4 \mathrm{G}$, and ideally the lowest for multimedia mHealth content delivery being 3G. However, mHealth is not limited to high quality signal strength and bandwidth, as services can be offered in various formats all with varying demands on communication bandwidth. At the lowest end of the spectrum simple Short Message Service (SMS) text based messages can be delivered with health information, alerts, and reminders. At the high end of the spectrum, providers can offer good quality multimedia information and service delivery. The associated cost being the requirement of a quality $4 \mathrm{G}$ telecommunication connection or high bandwidth WiFi connection for the mobile device.
For the mobile device itself, service delivery options are dependent on the features and technologies contained in the user's device. Legacy mobile phones are able to receive the base end of the services through the aforementioned SMS services. Preferably, however, users of mHealth systems would have access to and use of the ever-increasing array of smart phones and devices. These smart devices offer not only an upgrade to MMS (multimedia message service) but also the ability to access the Internet for multimedia rich web applications. Further, they can also download device specific native applications to be able to receive high quality multimedia services optimized to the device at hand. In addition, smart phones and modern tablet devices offer larger screens, of generally higher resolutions that afford more engaging and rewarding user experiences during the service delivery. While there are some limitations such as signal strength, battery recharge frequency, cost of device, and general maintenance, the mobile application platform offers a very effective medium for delivering health services. As such, we have seen over the past decade now a vast increase of applications both in the commercial and research domains.

It may appear the market and research areas for eHealth mobile application innovation are saturated. However, there are still hosts of niche areas that are in desperate need of such technological based health services. In particular, in the Australian environment there is an urgent need of eHealth/mHealth Information and Communication Technologies to assist the Indigenous people and their respective communities. Due to the vast geographical area of Australia, with many remote communities and general barren landscapes eHealth and its subclass of mHealth services have provided much needed assistance to the people populating these regions. In particular, studies have found that for those inclined to a nomadic culture that in contemporary times a mobile phone is one of the few personal items that remain in their possession. As such, while mobile reception is not always ideal in remote areas of Australia, a mobile device is proving an effective means for delivering health information and services, along with raising awareness of how to maintain both physical and mental health.

One such novel mHealth initiative is detailed in this paper. The StayinOnTrack [1] project is a multimedia ICT mHealth platform for the delivery of technology based physical and 
mental health support. The work presented in this paper focuses on the technological elements of the research project in question. The initial medical/health domain research contributions are published in [2]. As the research is a cross disciplinary collaboration, it represents the new successful convergence of previously disjointed academic workspaces, facilitated by the rapidly evolving technological landscape. The remainder of the paper is structured as follows: the following section provides background and related work; after which section 3 provides details of the conceptual design and framework the research produced; pilot implementation, testing and results are presented in section 4; while a conclusion and future work features in section 5. References are listed as the final section of the paper.

\section{BACKGROUND AND RELATED WORK}

A recent World Bank report [3] states that approximately $75 \%$ of the world's population has access to a mobile phone, and as of 2013 there are over 6 billion mobile phone service subscribers. With an increasing saturation of Information and Communication Technology (ICT) coverage and use, coupled with the portable nature of mobile technology, its use for providing Health Services is a given [4]. While technically a sub-domain of the eHealth field, mHealth is now frequently leading the way in innovative use of ICT for health provision. The authors in [5] provide a detailed look at the evolution of mHealth and summarize by stating that currently most mHealth solutions focus on interventions for chronic conditions. An extensive systematic review protocol is provided in [6], where the authors highlight the fact that to date little work has been done to evaluate the effectiveness of mHealth technologies. Further, the authors in [7] try to address validation issues by recommending innovative research designs such as multifactorial strategies, randomized controlled trials, and data farming.

Literature more closely aligned to the authors research project detailed in this paper fall under mental health and family 'action' (covering new fathers and parenting). As such, the authors in [8] and [9] provide two effective literature reviews covering important background research relevant to mobile application design, implementation, and testing for use in Mental Health. Further, the authors in [10] look at the use of mHealth technologies in clinical practice of behavioral health care. Research related to this papers work are provided in [11] and [12] where mHealth technologies are used in remote settings are discussed. A very encouraging study is provided in [13] which found $97 \%$ patients had a smart phone and $50 \%$ had an interest in using it daily to monitor mental health. Like our own work, the authors in [14] also looked at the effective use of social media integration with mHealth services [14]. Also of importance is ensuring the participants use the technologies in the study, and as such support for mobile phone use is provided in [15].

Lastly related works in mHealth for fathers provided valuable information in the technological development of our own work. The need to understand what fathers need and would use in terms of a mobile health application is very important. Useful perspectives were provided in [16 - 18], that used in conjunction with our own surveys and user feedback provided essential background information of Software Application design for our target user group.

\section{CONCEPTUAL DESIGN AND FRAMEWORK}

The project represents a cross disciplinary collaboration between health and engineering Faculties at the University of Newcastle (UoN). Specifically, the initial concept was devised through a group of leading family health academics from the UoN Family Action Centre [19]. As the conceptual idea and deliverable involved the use of ICT collaboration was sought from the lead author of this paper from the School of Electrical Engineering and Computing. From there the project gained momentum and conceptual design rapidly evolved to become a full-fledged merging of health services delivered through an ICT medium. That is, the research and project was quickly classified under an eHealth initiative. Fortunately, due to the high demand and urgent need of services to support the indigenous population the project was well received and garnered a wealth of support and attention.

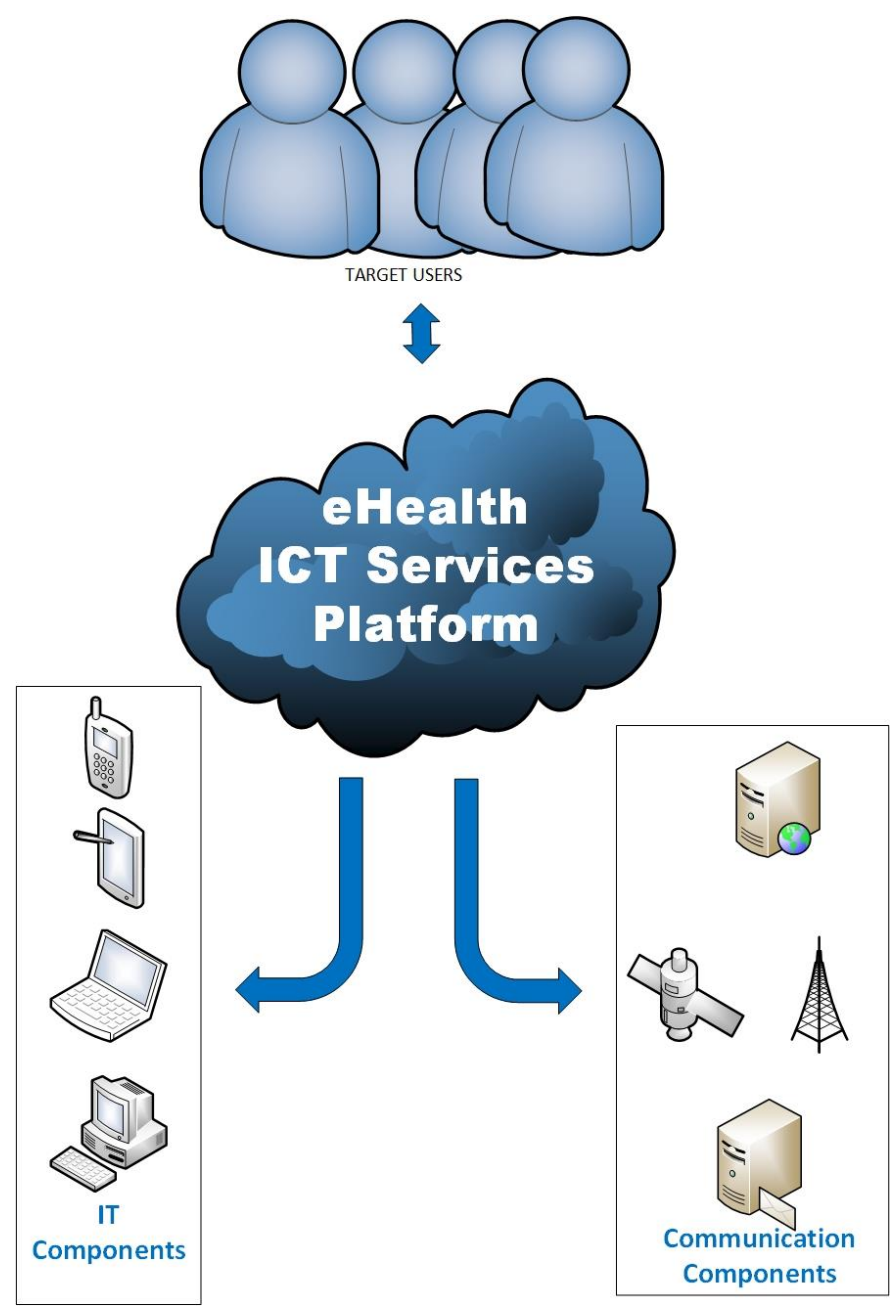

Figure 1. Required ICT features for conceptual artefact

The next phase of evolving the conceptual design included integrating both the research objectives from both a health and IT perspective, while also being able to produce a viable and usable artefact that was comparable to a commercial 
equivalent. This has long been a source of contention within the academic world especially from an IT perspective, and continues to be to this day. How does an IT\Computer Science academic justify his work on such projects and as those common in joint cross discipline research projects? That is the burning issue of: "How does one's contribution be seen as research rather than simply IT Support and Development?". However, this is a much to larger an issue to deal with here, but mention is made to highlight the fact that collaborators on the project were considerate to affording academic merit and contribution from the IT domain.
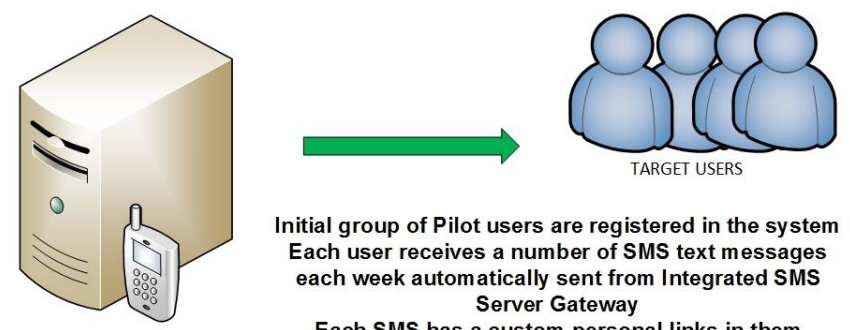

Initial group of Pilot users are registered in the system Each user receives a number of SMS text messages each week automatically sent from Integrated SMS Server Gateway

Each SMS has a custom personal links in them

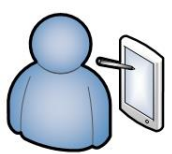

The user clicks the embedded link in SMS They are taken to a Web Application to provide feedback on their ' $m$ ental state' (mood)
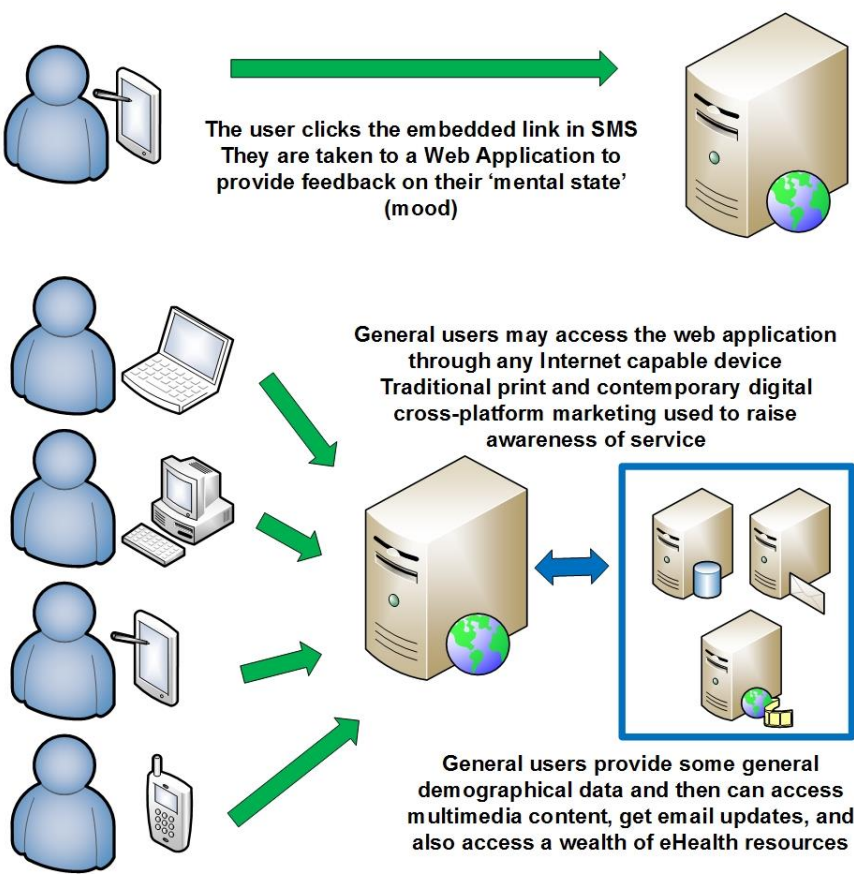

General users may access the web application through any Internet capable device through any Internet capable device
Traditional print and contem porary digital cross-platform marketing used to raise awareness of service

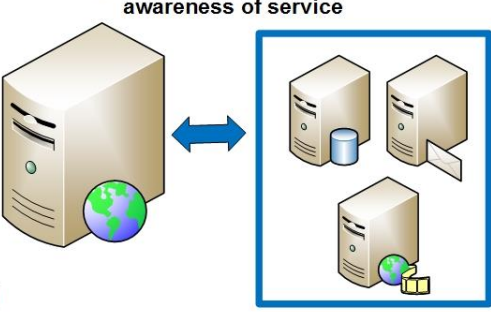

General users provide some general demographical data and then can access multimedia content, get email updates, and multimedia content, get email updates, and
also access a wealth of eHealth resources

Figure 2. Final conceptual design model

As the target demographic was already in place: young indigenous fathers the next step was to design and optimize the resulting artifact to best meet the needs, lifestyle, and capabilities of the desired user group. As such a mobile platform was deemed most appropriate and preferable, in addition to parallel standard web application interface design to accommodate all required access mediums (mobile, tablet, laptop, and desktop). With the IT dimension in place the communication formats needed to be defined. It was determined that due to the preferred mobile platform, then an initial point of contact along with follow up communications should be SMS based. The reasons being that they only require limited bandwidth to work, and also a baseline mobile device to access. In addition, where available emails will be used to provide enhanced multi-media communications, while a web based mobile application will store the bulk of the features, knowledge and processing requirements. Figure 1 displays the various conceptual design elements that were deemed necessary.

As the conceptual design continued to evolve it was decided to integrate video messages into the web application. In addition, a few other features were deemed necessary to satisfy other research objectives. These included: collection of demographical personal details, tracking of user browsing through click collection, and an interactive feature to capture the mental state or 'mood' of the user. The initial conceptual model involved the use of SMS messages containing personal custom links, a set of web pages to collect the user 'mood' then an automated email feature that triggered based on the 'mood' entered. As such the infrastructure hardware and software requirements needed to be expanded to not only include a web server, but also a database server, an SMS scheduling and sending service, and also automated email generation and messaging capabilities from the web application. The final conceptual design model that was the basis for a pilot implementation as shown in Figure 2.

\section{PROTOTYPE, IMPLEMENTATION AND RESUltS}

Like many research projects, the common process is to perform a validation study, or in ICT terms the testing and evaluation of a prototype. Once the validation study (in this project an integral part of that research work is the ICT Artefact discussed in this paper is successfully completed, then often the next stage for health related projects is a Randomized Controlled Trial. The first part of this section details the prototype, and is followed by explaining the 'final' implemented eHealth platform that has gone on to be used in a number of further RCT's and eHealth research projects [20].

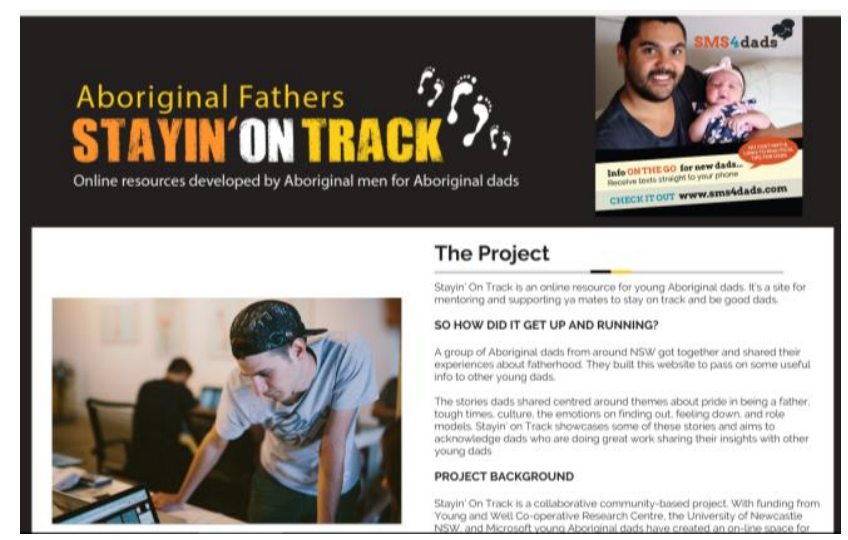

Figure 3. Home page with project overview

Due in part to the generous support provided by Microsoft Australia, the prototype is implemented using Microsoft Web and Data Technologies. With contributions of Microsoft Mobile phones provided for all the initial pilot group users, as it made sense to build the Web Application using Asp.Net/C\#.Net to ensure the highest compatibility with the hardware devices. Additionally, a dedicated MS SQL 
Server was required for the database backend, MS Internet Information Server (IIS) for the web server platform, and a dedicated Microsoft Virtual Server to host the web application. The entire ICT infrastructure is configured, maintained and located 'in house'. As such, the rigorous cyber security environment provided by University of Newcastle protects everything. Such a hosting environment also provided ample bandwidth to support the streaming of the multimedia components of the final web application.

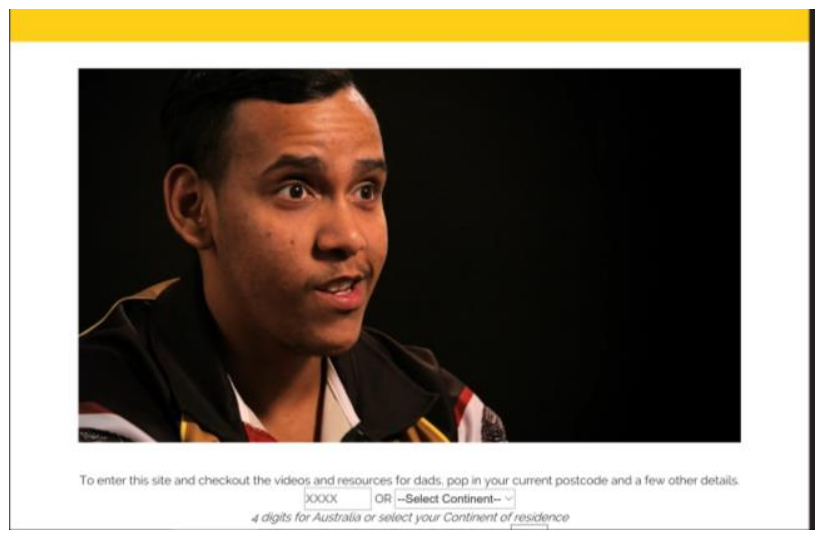

Figure 4. Landing page multimedia video

Architecturally, the web application is a mixture of entity framework and traditional n-tier implementations. A mixed approach to the coding architecture allowed various developers to work on separate components of the application in parallel, while also allowing rapid application development targeted at specific functionalities of the web application. This reduced development life cycles, while also allowing a flexible and adaptive approach to constantly changing user requirements. An important point to note is that much of the User Layer for the application evolved through user driven design. That is, a number of indigenous elders were constantly reviewing and refining the design and multimedia elements of the application to ensure it provided a culturally sensitive targeted user experience. Further, the first group of pilot users have also provided useful feedback on the application, which is being used as input to continue the evolution of the eHealth service.

The current live production ICT solution (StayinOnTrack) is best described as delivering multimedia and informational eHealth support to new young indigenous fathers through digital technologies. The Project Overview and welcome page is shown in Figure 3. The list of major features the application provides are as follows:

1) Visitor to the web application are provided an initial short film of young aboriginal fathers talking about their experiences and the StayinOnTrack application (fig 4).

2) Links to numerous contextual informational resources including additional multimedia content such as videos (fig 5) and interactive presentations, help support lines and forums, and online documents and information (fig 6) to help new fathers.

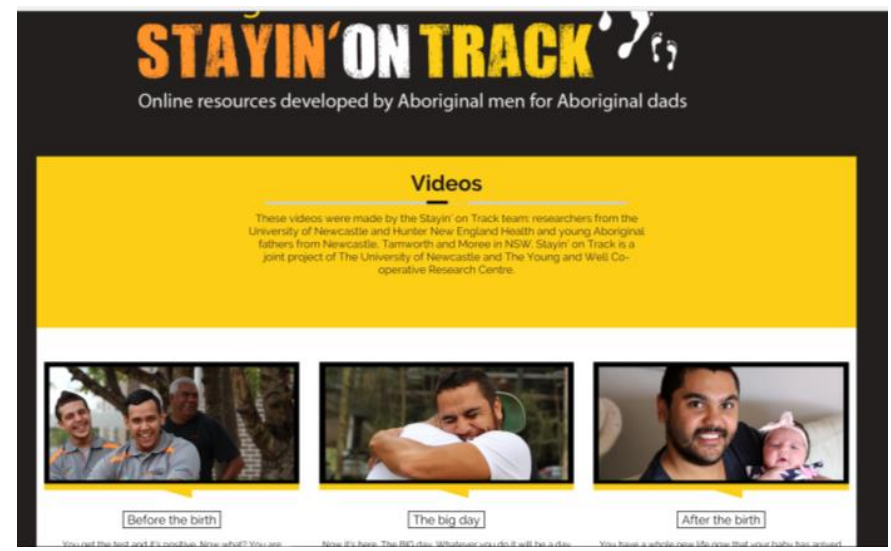

Figure 5. Additional 'Dad' streaming videos

3) A SMS prompted Mood Tracker module within the StayinOnTrack application. Daily, registered fathers in the program are sent various automated 'mental health' related SMS with embedded links. The links take the user to a Mood Tracker application where they can select one of five moods on scale: Solid-Deadly-OK-Mad-Low (fig $\mathrm{x}$ ). At this time if any user records more than two consecutive "Lows" they are immediately contacted by one of the project teams mental health indigenous elders to have a chat with the user in potential trouble.

4) Other standard informational web site pages such as details about the project and project team, contact details, funding details, and acknowledgements.
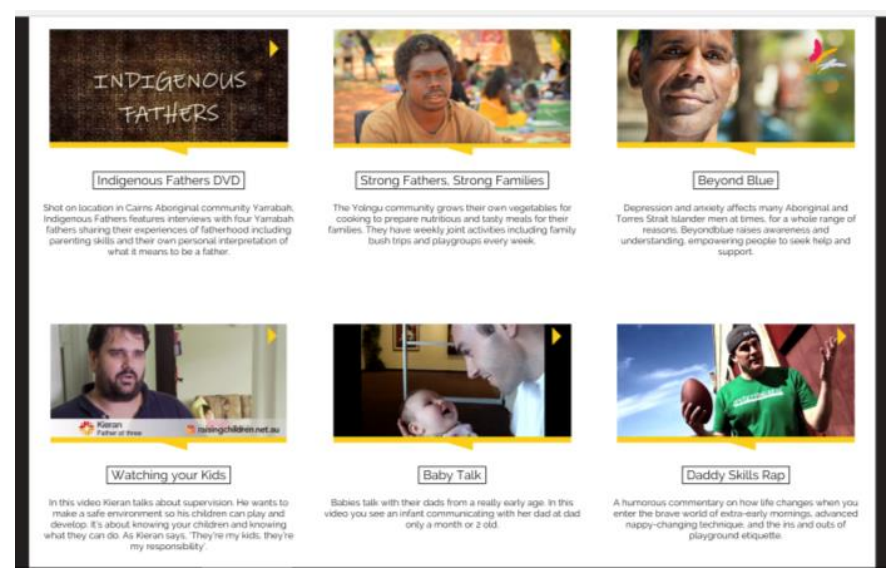

Figure 6. 'Dad' information links and multimedia resources

At time of writing this paper it has been close to 18 months since the prototype and production version of StayinOnTrack has been live. The project is deemed an overwhelming success based on participant surveys, feedback and peer review. Primary highlights from the user's feedback indicate the multimedia elements of the application were great and very engaging. Further, the mobile and accessible nature of the application were seen as key elements to its success. However, the most promising and innovative element of the project has been the Mood Tracker. The integration of SMS and email contact points to 'check in' with participants to not only gauge 
their mental health state, but also show support is there, was by far the biggest success of the project from both a Health and ICT perspective. The project has been another great example of the successful merging and collaboration of the ICT and health domains. The result has been a very innovative eHealth platform that has been utilized as the foundation for numerous ongoing other eHealth research initiatives [21].

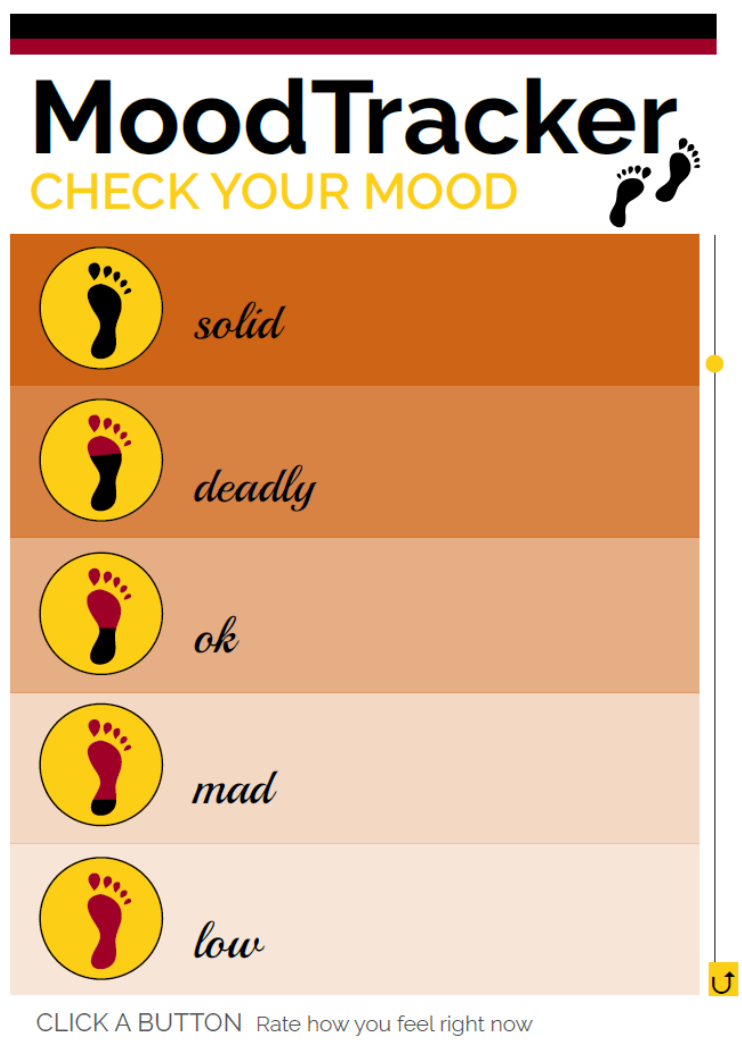

Figure 7. Mood Tracker Module

\section{CONCLUSION AND FUTURE WORK}

The StayinOnTrack research initiative has proved to be a very successful project, representing a sound collaboration between the Heath and ICT research domains, now commonly referred to as eHealth. Provided here in, are the ICT details of the StayinOnTrack software application, which more specifically is classified as a mHealth (mobile health) service. That is, the web-based application has been developed with mobile devices as the target user device access medium, and delivers rich family action and mental health multimedia content, services and support to these digital platforms. In large geographical locations such as Australia technology is becoming an ever-increasing asset in providing quality accessible health services to remote people and their respective communities. StayinOnTrack is one such solution that provides young indigenous fathers with mental health support, multimedia informational resources, and access to peers and elders alike for more personalized care on being a new father.
Results to date are conclusive in that StayinOnTrack has been a success and well received by the partipants. The web application and project continues to function providing eHealth services to an ever-increasing user base. Based on its success many of the technological elements first developed and tested within the application have been used in new projects and we plan to evolve them further for more future projects. The next evolution of the Mood Tracker for example will include a tree like response structure rather than a single pick 1 of 5 options response. It will have increased SMS activity based on the different responses procided by participants at each successive level of the mood tracker tree. Additionally, as evidenced by our other current projects the eHealth platform has been extended to provide similar services to new mothers, and a more generalised version for fathers of all demographics. In short, the eHealth multimedia solution we have developed continues to evolve and garner recent international attention.

\section{REFERENCES}

[1] Aboriginal Fathers StayinOnTrack - Online resources developed by Aboriginal men for Aboriginal Fathers. Available: https://www.stayinontrack.com/. Accessed 20th of February 2017.

[2] Fletcher, Richard, et al., "StayinOnTrack, Supporting young Aboriginal fathers through a user developed website," Australian Association for Infant Mental Health Conference 2015, October, 2015.

[3] ICD (2012) Information and communications for development: maximizing mobile. Available: http://www.worldbank.org/ict/IC4D2012. Accessed 17 February 2017.

[4] Kay, Misha, Jonathan Santos, and Marina Takane. "mHealth: New horizons for health through mobile technologies." World Health Organization 3 (2011): 66-71.

[5] Fiordelli, Maddalena, Nicola Diviani, and Peter J. Schulz. "Mapping mHealth research: a decade of evolution." Journal of medical Internet research 15.5 (2013): e95.

[6] Free, Caroline, et al. "The effectiveness of M-health technologies for improving health and health services: a systematic review protocol." BMC research notes 3.1 (2010): 250.

[7] Tomlinson, Mark, et al. "Scaling up mHealth: where is the evidence?." PLoS Med 10.2 (2013): e1001382.

[8] Naslund, John A., et al. "Emerging mHealth and eHealth interventions for serious mental illness: a review of the literature." Journal of Mental Health 24.5 (2015): 321-332.

[9] Donker, Tara, et al. "Smartphones for smarter delivery of mental health programs: a systematic review." Journal of medical Internet research 15.11 (2013): e247.

[10] Luxton, David D., et al. "mHealth for mental health: Integrating smartphone technology in behavioral healthcare." Professional Psychology: Research and Practice 42.6 (2011): 505.

[11] Akter, Shahriar, and Pradeep Ray. "mHealth-an ultimate platform to serve the unserved." Yearb Med Inform 2010 (2010): 94-100.

[12] Lori, Jody R., et al. "Cell phones to collect pregnancy data from remote areas in Liberia." Journal of Nursing Scholarship 44.3 (2012): 294-301.

[13] Torous, John, Rohn Friedman, and Matcheri Keshavan. "Smartphone ownership and interest in mobile applications to monitor symptoms of mental health conditions." JMIR mHealth and uHealth 2.1 (2014): e2.

[14] Brusse, Carl, et al. "Social media and mobile apps for health promotion in Australian Indigenous populations: scoping review." Journal of medical Internet research 16.12 (2014): e280.

[15] McCallum, Gabrielle B., et al. "Mobile phones support adherence and retention of indigenous participants in a randomised controlled trial: strategies and lessons learnt." BMC public health 14.1 (2014): 622. 
[16] Kim, Hyung Nam, et al. "Use of Social Media by Fathers of Premature Infants." The Journal of Perinatal \& Neonatal Nursing 30.4 (2016): 359366.

[17] Umali, Elaine, Judith McCool, and Robyn Whittaker. "Possibilities and expectations for mHealth in the Pacific Islands: insights from key informants." JMIR mHealth and uHealth 4.1 (2016).

[18] Albanese-O'Neill, Anastasia, et al. "Educational Needs and Technological Preferences of Fathers of Youth With Type 1 Diabetes." The Diabetes Educator 42.2 (2016): 209-219.
[19] The Family Action. Available: https://www.newcastle.edu.au/researchand-innovation/centre/fac/about-us. Accessed Accessed 20th of February 2017.

[20] Fletcher, Richard, et al. "SMS4dads: Providing information and support to new fathers through mobile phones-a pilot study." Advances in Mental Health (2016): 1-11.

[21] SMS4Dads\&Mums - info for new mums and dads, Available: https://www.sms4dnm.com/ , accessed 20th Feburary, 2017. 